Icarus

\title{
The Precession Constant and its Long-Term Variation --Manuscript Draft--
}

Manuscript Number:

Article Type:

Keywords:

Corresponding Author:

First Author:

Order of Authors:

Abstract:

\section{Suggested Reviewers:}

ICARUS-D-20-00066R1

Letter

Precession constant; dynamical flattening and Earth rotation; mantle convection; glacial isostatic adjustment; modern climate change

Jerry X Mitrovica

Harvard University

UNITED STATES

Siavash Ghelichkhan, $\mathrm{PhD}$

Siavash Ghelichkhan, PhD

Jocelyn J Fuentes, PhD (April 6, 2020)

Mark J Hoggard, PhD

Fred D Richards, PhD

Jerry X Mitrovica

The dynamical flattening of the Earth, $\mathrm{H}$, related to the precession constant, is a fundamental astro-geodetic parameter that appears in studies of the Earth's rotation and orbital evolution. We present numerical predictions and observations of the variation in $\mathrm{H}$ over time scales ranging from tens of millions of years to decades. The geophysical processes controlling this variation include solid-state convection in the rocky mantle of the Earth that drives plate tectonics, isostatic adjustments due to ice age loading, and ice-ocean mass transfer linked to modern global climate change. The time dependence of $\mathrm{H}$ is complex and non-linear, and thus, in contrast to previous suggestions, cannot be captured by a constant rate parameter.

Isamu Matsuyama, $\mathrm{PhD}$

Associate Professor, University of Arizona

isa@lpl.arizona.edu

Expert in planetary rotational dynamics

Sabine Stanley, PhD

Bloomberg Distinguished Professor, Johns Hopkins University

sabine@jhu.edu

Expert in planetary structure, rotation and dynamics; geophysicist

David J Stevenson, PhD

Marvin L Goldberger Professor of Planetary Science, California Institute of Technology djs@gps.caltech.edu

Expert in planetary and Earth science

Michael Manga, PhD

Garniss H. Curtis Endowed Department Chair, University of California, Berkeley manga@seismo.berkeley.edu

Expert in planetary dynamics, rotation and evolution

Veronique M Dehant, PhD

Head of Service, Observatoire Royal de Belgique

veronique.dehant@oma.be

Expert in planetary rotation and sciences

Opposed Reviewers:

W Richard Peltier, PhD

Professor, University of Toronto

wrp@physics.utoronto.ca

Prof. Peltier has a long-standing animosity toward my group and my students. He would be incapable of an objective review. My opposition to him seeing any of my work before publication is absolute. If the journal were to consider sending him the manuscript - which is unlikely given that he is not an expert - I would ask that the paper 
be withdrawn.

Response to Reviewers:

Powered by Editorial Manager ${ }^{\circledR}$ and ProduXion Manager ${ }^{\circledR}$ from Aries Systems Corporation 


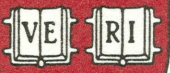

|TAS

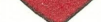

\author{
HARVARD UNIVERSITY \\ DEPARTMENT OF EARTH AND PLANETARY SCIENCES \\ 20 OXFORD ST. \\ CAMBRIDGE, MA 02138 \\ TEL. (617) 496-2732 FAX. (617) 495-8893
}

March 15, 2020

Dear Editor:

We have submitted a revised version of the manuscript "The Precession Constant and its Long-Term Variation" for your continued consideration as a Letter to Icarus. The manuscript is co-authored by Siavash Ghelichkhan, Jocelyn Fuentes, Mark J. Hoggard, Fred D. Richards and Jerry X Mitrovica (corresponding author). We note that the order of the first and second listed authors of the original manuscript has been switched in the revised version. This reflects the effort required to revise the article and the change has been fully agreed upon by all authors.

As you will note from the response letter attached to this submission, we have comprehensively addressed all of the comments raised in the single, positive review that we received. We believe that these changes have strengthened the manuscript and we genuinely enjoyed considering and addressing the interesting issues that the reviewer raised.

Given the combination of astronomical and geological issues discussed in the manuscript, we continue to believe that Icarus is the ideal journal in which to present our results.

We look forward to hearing from you in due course,

Sincerely,

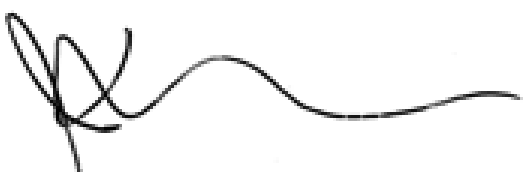

Jerry X. Mitrovica

Frank B. Baird, Jr., Professor of Science 


\section{Dear Editor:}

We have submitted a revised version of the manuscript "The Precession Constant and its Long-Term Variation" (ICARUS-D-20-00066). The revised manuscript received a single, positive review that raised three points of concern. In the material below, we respond comprehensively to each point, and indicate the associated changes to the text. The reviewer's comments appear in blue and our responses are in black - text quoted from the original or revised manuscript is indented. We note that the revised manuscript switches the order of the first and second authors. All authors have agreed on this change, which reflects the work that was performed in revising the manuscript.

Reviewer \#1: The paper presents more realistic computations of the Earth's precession constant variations, which has important implications for precession and obliquity. Given these important implications and the significant differences between the calculations presented in the paper and previous models assuming a single rate term, this paper would be a valuable addition. However, some issues need to be addressed before I can recommend it paper for publication.

We thank the reviewer for the positive assessment of the novelty and importance of our manuscript.

We recognized after reading the review that our original manuscript included sloppy terminology that may have played a role in some of the reviewer's comments. Our manuscript is concerned with changes in the ellipticity or flattening of the Earth and in the text of the manuscript we used these terms interchangeably with "precession constant". The latter formally refers to the annual rate of precession of the equinoxes, while the former is a measure of the flattening (or ellipticity) of the Earth. The precession constant is controlled by the dynamical flattening (or ellipticity), but in a formal sense they are not the same thing. It has been common in the literature to equate the two terms, but to do so is to be imprecise - as we were.

A variety of language is also used in the literature to distinguish the terms, for example Burša et al. (2008) write: "The coefficient associated with the precession constant, $H$, which is often called dynamical ellipticity or dynamical flattening ...". The subtle distinction between the two is important and we have revised the text to make sure we do not obscure the distinction.

\section{Major comments:}

Eq. 2 is only valid for deformations that do not involve a spherical component of degree0 (see the paragraph below Eq. 9 in Rochester and Smylie). Therefore, it is only valid for an incompressible interior unless the forcings responsible for the deformations do not involve degree- 0 , which is difficult to justify for mantle convection, ice age 
perturbations, and recent melting of glaciers. The incompressible interior assumption needs to be justified and stated clearly.

The reviewer is correct to point out that a degree-0 term will impact the inertia tensor of the Earth - there are many examples in our previous work on rotational dynamics of terrestrial planets where we make this connection explicit (e.g., Matsuyama et al., JGR, v. 111, 2006; equation 3). The contribution of the degree- 0 term is commonly left out of the expression for the perturbation in the inertia tensor (as in equation 2 of the original manuscript - see, for example, Burša et al., 2008) because it impacts all three principal axes $(A, B$ and $C)$ identically. That is, while a degree-0 volume term would introduce an inequality in equation (2), it would have relatively small impact on our expressions for the dynamical flattening or ellipticity (or the results based upon them).

Before addressing this issue in more quantitative terms, we first respond to the reviewer's argument that "Therefore, it is only valid for an incompressible interior unless the forcings responsible for the deformations do not involve degree-0, which is difficult to justify for mantle convection, ice age perturbations, and recent melting of glaciers". In fact, the forcings associated with ice age perturbations and the recent melting of glaciers have no degree- 0 component because these processes conserve mass. That is, the surface mass load driving these signals includes changes in both ice and sea level components - and conserving the total surface mass represents a key constraint in the calculations. As we wrote on Line 109 of the manuscript:

Our prediction of perturbations to the precession constant (Figure 1B) is generated using a theory of ice age dynamics that involves a gravitationally self-consistent treatment of sea level changes (Kendall et al., 2005).

We have revised this sentence to read (line 112):

Our prediction of perturbations to the precession constant (Figure 1B) is generated using a theory of ice age dynamics that involves a gravitationally self-consistent treatment of sea level changes constrained to conserve the total (ice plus ocean) surface mass (Kendall et al., 2005).

All such calculations ensure that the change in ice-plus-ocean mass is identically zero.

In regard to mantle convection, the situation is more nuanced. The reviewer writes:

"(equation 2) is only valid for an incompressible interior". First, the equations governing mantle convection that we solve incorporate compressibility in the form of the anelastic liquid approximation, which ensures a proper treatment of compressibility effects arising from the orders of magnitude increase in pressure with depth in the mantle, as indicated within the original (and revised) Appendix: 
Once the temporal evolution of the mantle flow field has been successfully reconstructed, we calculate the time history of dynamic ellipticity, $H$. For this purpose, we solve the governing, coupled system of Stokes and Poisson's equations using an instantaneous flow methodology that includes the effects of self-gravitation and compressibility and assumes a free-slip (no tangential stress) boundary condition (Corrieu et al., 1995).

Furthermore, our formalism for reconstruction of mantle structure comes from an explicit treatment of compressibility effects in the geodynamic adjoint equations (Ghelichkhan \& Bunge 2016, cited in the Appendix). So, our governing equations include compressibility. However, this does not mean that the Earth is subject to net volume changes because an increase in density in one mantle location is balanced by a decrease elsewhere. Nevertheless, the main process that will drive a degree-0 change in the shape of the Earth is secular cooling, a process that is not included in our mantle convection simulations and is ignored in our equation (2). How big is this term?

To answer that question, we can augment equation (3) of the original manuscript (corrected for the sign error noted below) to the form that accounts for a degree-0 perturbation to the inertia tensor:

$\delta \mathrm{H}=\left[3 / 2-\mathrm{H}\left(1+\delta \mathrm{C}_{(0,0)} / \delta \mathrm{C}_{(2,0)}\right)\right] \delta \mathrm{C}_{(2,0)} / \mathrm{C}$

This expression differs from the equation in the manuscript through the addition of the term $\delta \mathbf{C}_{(0,0)} / \delta \mathbf{C}_{(2,0)}$.

How much has the Earth's radius changed with time due to cooling and thermal contraction? There is general consensus that the temperature of the mantle during the Archean Period was of the order of $100^{\circ} \mathrm{C}$ greater than today (Ganne \& Feng, Geochem. Geophys. Geosys., v. 18, 2017), or about $1^{\circ}$ over 50 Myr. A simple scaling analysis that equates the change in volume $(\delta \mathrm{V})$ due to a change in temperature $(\delta \mathrm{T})$ (i.e., $\delta \mathrm{V}=\mathrm{V} \alpha \delta \mathrm{T}$, where $\alpha$ is the coefficient of thermal expansion, $\sim 3 \times 10^{-5} \mathrm{~K}^{-1}$ ) with the change in volume due to a small change in radius $(\delta \mathrm{V}=3 \mathrm{~V} \delta \mathrm{r} / \mathrm{a}$, where $\mathrm{a}$ is the radius of the Earth), yields the following expression for the change in radius: $\delta r=\alpha \delta \mathrm{T} a / 3$. Plugging in the above numbers, yields a contraction of $\sim 65 \mathrm{~m}$ since $50 \mathrm{Ma}$. This agrees well with estimates shown in Tsuchiya et al. (Geosci. Frontiers., v. 4, 2013), $>80 \mathrm{~m}$ over the same time period and we will adopt this value for $\delta r$ due to contraction. Perturbing the expression for the moment of inertia of a sphere yields $\delta \mathrm{C}_{(0,0)}=(4 / 5) \mathrm{Me}$ a $\delta r$ (where $\delta r$ is a negative number, and $\mathrm{Me}_{\mathrm{e}}$ is the Earth's mass).

Next, we turn to the term $\delta \mathrm{C}_{(2,0)}$. We can simply look up this value from results of our convection simulation, but we can gain some physical insight by considering the change in the flattening, $f$, of the Earth (i.e., the difference in the equatorial and polar radius). From Figure $1 \mathrm{~A}$, the peak-to-peak perturbation to $\delta \mathrm{H} / \mathrm{H}$ reaches 0.0043 over the past 50 Myr. A paper by members of our group (Morrow et al., Geophys. J. Int., v. 191, 2012) 
derives the following relationship between the flattening and the perturbation $\delta \mathrm{H} / \mathrm{H}$ (see their equation 3): $f=1.06 \times 10^{4} \delta \mathrm{H} / \mathrm{H}$, and thus the result in our Figure $1 \mathrm{~A}$ is equivalent to a decrease in the flattening of $\sim 45 \mathrm{~m}$ over this same period. Perturbing the expression for the moment of inertia of an ellipsoid of revolution yields $\delta \mathrm{C}_{(2,0)}=(4 / 15)$ Meaf (where $f$ is a negative number). Thus, $\delta \mathrm{C}_{(0,0)} / \delta \mathrm{C}_{(2,0)} . \sim 5$, and $\mathrm{H}\left(1+\delta \mathrm{C}_{(0,0)} / \delta \mathrm{C}_{(2,0)}\right) \sim 6 \mathrm{H} \sim 0.02$ (which is $<$ that the leading term in the above equation, $3 / 2$ ). We conclude that ignoring the impact on $\delta \mathrm{H} / \mathrm{H}$ of thermal contraction introduces an error that is of order $1 \%$.

To address this issue in the manuscript, we have performed a series of revisions. First, above equation (2), we have changed the original text:

Perturbing Equation (1), and using the fact that the trace of the inertia tensor is invariant during deformation (e.g., Rochester and Smylie, 1974)

to read

Perturbing Equation (1), and using the fact that the trace of the inertia tensor is invariant during non-uniform deformation (e.g., Rochester and Smylie, 1974) ... (NB. we comment in Section 3 on the impact of uniform deformation on these equations)

Furthermore, we have added the following text as a penultimate paragraph to the Results section:

The calculations in Figure 1, since they are based on Equation (2), do not include the impact on the inertia tensor of a uniform, degree-0 deformation of the Earth. If we included this spatially uniform signal in the theory, Equation (3) would be revised to

$$
\delta \mathrm{H}=\left[3 / 2-\mathrm{H}\left(1+\delta \mathrm{C}_{(0,0)} / \delta \mathrm{C}_{(2,0)}\right)\right] \delta \mathrm{C}_{(2,0)} / \mathrm{C},
$$

where the subscript denotes the spherical harmonic degree and order of the structure contributing to the inertia perturbation. In our calculations of perturbations in $\delta \mathrm{H} / \mathrm{H}$ due to ice mass changes (Figure $1 \mathrm{~B}$ ), we include complementary sea level changes and the total mass of the surface load is conserved (i.e., it has no degree-0 component). The same must be true for the processes responsible for the observations that form the basis of Figure 1C. Thus, in these cases, there is no degree-0 deformation, $\delta \mathrm{C}_{(0,0)}=0$, and the above expression collapses to Equation (3). While our calculations of the perturbation to $\delta \mathrm{H} / \mathrm{H}$ driven by mantle convection adopt a compressible flow model, any changes in the volume of the Earth are negligible. However, these calculations do not include secular cooling and thermal contraction of the Earth. Estimates of this process suggest that the reduction in Earth radius over the past 50 Myr due to thermal contraction has been $\sim 80 \mathrm{~m}$ (Tsuchiya et al., 2013). Using this value, and the results in Figure $1 \mathrm{~A}$, yields the estimate 
$\delta \mathrm{C}_{(0,0)} / \delta \mathrm{C}_{(2,0)} \sim 5$, and $\mathrm{H}\left(1+\delta \mathrm{C}_{(0,0)} / \delta \mathrm{C}_{(2,0)}\right) \sim 6 \mathrm{H}$. This value is of order $1 \%$ of the leading term of $3 / 2$ in Equation (8) and neglecting it in adopting Equation (4) remains justified.

L60-61: The conservation of the Earth's rotational angular momentum needs to be justified. What is conserved is the angular momentum of the Earth-Moon system, which has contributions from Earth's rotational angular momentum and the orbit angular momentum. This seems important given that the authors are considering tidal dissipation perturbations to $\mathrm{dH} / \mathrm{dt}$.

In fact, in the original manuscript we did not consider tidal dissipation perturbations to the dynamical flattening in our Figure 1 (though it is mentioned in the Introduction). Nevertheless, the reviewer's comment regarding conservation of angular momentum is of course correct, but our text at this point in the manuscript was intended simply as a statement of the impact of the various processes that we are considering (mantle convection, ice age dynamics, modern ice melting) on the rotation rate - it was not intended as a suggestion that these processes are the only ones that can influence the rotation rate. In this regard, since none of these processes changed the rotation rate significantly over the longest time window we consider in the manuscript (50 Myr in Figure 1A), each process can be considered to have an independent effect on the rotation rate. Consider, as an example, the tidal dissipation process the reviewer is referring to. Geological records (e.g., Williams, Geophys. Res. Lett., v. 24, 1997) suggest that the rotation rate at $620 \mathrm{Ma}$ was $\sim 10 \%$ faster than today $\left(8 \times 10^{-5} \mathrm{rad} / \mathrm{s}\right.$ at $650 \mathrm{Ma}$ versus $7.3 \times 10^{-5} \mathrm{rad} / \mathrm{s}$ at present); assuming linearity, this would indicate a small reduction in the rotation rate of $<1 \%$ since $50 \mathrm{Ma}$. (The same upper bound would emerge if one used the present-day tidal dissipation rate to estimate the change in rotation rate at $50 \mathrm{Ma}$; see Laskar et al., Astron. Astrophys., v. 270, 1993.)

In any case, it is important to emphasize that Equation (5) of the original manuscript that the reviewer is pointing to has no bearing on the focus of our study - that is, predictions of $\delta \mathrm{H} / \mathrm{H}$ arising from mantle convection, ice age dynamics and modern melting of ice sheets and glaciers - and we have thus deleted it from the manuscript.

L68-71. The connection between the precession constant and the frequency of a perturbations is not clear. This should be explained in more detail, including an equation relating the precession constant and the frequency of the perturbation.

This relationship is a standard proportionality in the case when the precession frequency is out of resonance with any gravitational forcing in the solar system (see, e.g., Williams, Astron. J., v. 108, 1994). That publication, together with Laskar et al. (1993) show the relevant equations and we have added citations to both of them at this point in the text. 


\section{Minor comments:}

Writing Eq. 1 also explicitly in terms of $\mathrm{J} 2$ would help readers make the connection between the precession constant and $\mathrm{J} 2$, especially for those who are not familiar with the definition of $\mathrm{J} 2$.

To address this comment, we have revised the sentence above the original Equation 7 (now Equation 6):

Comparing equations (1) and (6) yields the following relationship between perturbations in $\mathrm{H}$ and $\mathrm{J}_{2}$

to read:

Combining equations (1) and (5) yield the following relationship,

$$
\mathrm{H}=\mathrm{Me}_{\mathrm{e}} \mathrm{a}^{2} \mathrm{C} \mathrm{J}_{2} .
$$

L45-48: I'm assuming that tidal dissipation results in a perturbation in $\mathrm{dH} / \mathrm{dt}$ due to the perturbation on the rotation rate? It might be worth discussing the connection between tidal dissipation and $\mathrm{dH} / \mathrm{dt}$ in more detail.

We agree, but feel that this discussion is best suited to the end of the Results section where we can point out that tidal dissipation, which is not modeled, also impacts the dynamical flattening. In particular, we have added the following as a final paragraph of that section:

The results in Figure 1 do not include the impact on the dynamical flattening of an additional process mentioned in the introductory section, namely, tidal dissipation. The present level of tidal dissipation is slowing the Earth's rotation at a rate of $d \Omega / d t$ $/ \Omega=8.8 \times 10^{-18} \mathrm{~s}^{-1}$ (e.g., Quinn et al., 1991) and the dynamical flattening will be approximately proportional to $\Omega^{2}$. While the variation of tidal dissipation over time is uncertain, any effort to estimate the total change in dynamical flattening from all geophysical processes must include this contribution.

Eq. 3, the term in the square brackets should be $[3 / 2-\mathrm{H}]$ instead of $[3 / 2+\mathrm{H}]$

Thank you for catching this typo. We have made the correction.

Eq. 7, the right-hand-side is missing a term associated with the perturbation of $\mathrm{C}$. After some algebra and using $\mathrm{H}<<1$, it does reduce to the expression in Eq. 7 but this should be explained in more detail. In particular, the same approximation used in Eq. 4 is being 
used here.

Agreed. We should have made the underlying approximation clear in the original manuscript. We have revised the following text that appeared above the original Equation 7

Comparing equations (1) and (6) yields the following relationship between perturbations in $\mathrm{H}$ and $\mathrm{J}_{2}$

to read:

Equations (1) and (5) yield the following relationship,

$$
\mathrm{H}=\mathrm{Me}^{2} / \mathrm{C} \mathrm{J}_{2} \text {. }
$$

Taking the first variation of this expression, and once again using the fact that $\mathrm{H} \ll$ 1 , yields

Figure $1 \mathrm{~A}$. The authors could discuss the physics behind the change in the sign of $\mathrm{dH} / \mathrm{dt}$ around $20 \mathrm{Ma}$. Is there a major change in the convection perturbations at this time?

To investigate the physics of changes in dynamic flattening, we examined the so-called "dynamic geoid response functions", which relate mantle structure to the corresponding gravitational equipotential figure of the Earth. Dynamic response functions encapsulate the gravitational signal of both internal density anomalies and the associated viscous boundary deflections of the CMB and surface. At the longest wavelengths (e.g., degree 2 ), these functions are positive in the upper mantle, but negative in the lower $1000 \mathrm{~km}$ of the mantle.

Current seismic tomography models and inferences of present-day mantle structure based upon model reconstructions of plate tectonics suggest the persistence of degreetwo buoyancy distribution in the deep mantle, with two slow velocity anomalies beneath Africa and the Pacific Ocean that have been girdled by a continuous subduction system since the breakup of Pangea. In the transition zone, density anomalies are generally found to be anti-correlated with this deeper mantle structure.

A key implication of these theoretical and observational arguments is that present-day transition zone anomalies and deep mantle anomalies are both expected to constructively contribute to the geoid signal (due to the sensitivity kernel switching sign). Thus, an increase in dynamic flattening would be expected if either transition zone or deep mantle degree-2, order-0 anomalies have increase in amplitude through time, and vice versa. These contributions vary depending on the growth of Rayleigh-Taylor instabilities at the base of the mantle (the location of plume inception), and episodic motion of subducting slabs. Our analysis indicates that the general increase in $\delta \mathrm{H} / \mathrm{H}$ up 
to $20 \mathrm{Ma}$ is caused by an increase in the degree-2, order-0 component of density anomalies in the deep mantle, accompanied by a further decrease in the transition zone (i.e. both anomalies grow up to this point). From $20 \mathrm{Ma}$ to present, the transition zone anomalies begin to decrease in amplitude as slab material starts to sink beneath the transition zone, and this causes $\delta \mathrm{H} / \mathrm{H}$ to begin to decrease towards the present day. We have summarized this rather technical explanation by adding the following text to the manuscript (line 98):

Our investigation of the evolving mantle heterogeneity in the adjoint model indicates that the increase in $\$$ ldelta $\mathrm{H} / \mathrm{H} \$$ from $50 \mathrm{Ma}$ to $20 \mathrm{Ma}$ is driven by an increase in the amplitude of long-wavelength density anomalies at the base of the upper mantle (the so-called transition zone) and the base of the lower mantle (i.e., above the fluid outer core). The subsequent change in trend reflects a progressive weakening of the transition zone signal after $20 \mathrm{Ma}$.

Fig. 1C. The connection between the negative $\mathrm{dH} / \mathrm{dt}$ and melting from polar ice sheets could be made clearer by discussing the perturbations to the moments of inertia and J2.

Agreed. After the following text on line 129:

Finally, we turn our attention to recent variations in the dynamical flattening on decadal time scales. Figure $1 \mathrm{C}$ shows the observed change in $H$ across the satellite period, relative to 2012, derived from the results of Cheng et al. (2013). As discussed earlier, a change in the trend of the $\delta \mathrm{H} / \mathrm{H}_{0}$ time series, or equivalently $\mathrm{J}_{2}$ (Equation 5), took place around the year 1990.

we have added:

Prior to that date, the trend is dominated by the above-noted reduction in oblateness (and polar moment of inertia) since $\sim 6$ ka driven by the ongoing effects of the ice age. This trend continues after 1990, but the onset of significant melting of ice sheets at that time contributes an increase in oblateness (as ice melts near the poles and mass redistributes toward lower latitudes); the net signal is characterized by a reduced trend (i.e., the magnitude of $\mathrm{dH} / \mathrm{dt}$ and $\mathrm{dJ} / 2 / \mathrm{dt}$ decreases).

Once again, we thank the reviewer for raising the above issues. The associated revisions have improved the manuscript and we genuinely enjoyed the process of considering and addressing the very interesting points raised in the review. 
The dynamical flattening $H$ appears in studies of Earth rotation and orbital evolution Modern climate, ice age dynamics and mantle convective flow all drive variations in $H$ We quantify this variability using modern observations and new geophysical modeling The variation in $H$ is highly non-linear on time scales ranging from decades to $10^{8} \mathrm{yr}$ 


\title{
The Precession Constant and its Long-Term Variation
}

\author{
Siavash Ghelichkhan $^{1}$, Jocelyn J. Fuentes ${ }^{2}$, Mark J. Hoggard ${ }^{2,3}$, Fred D. Richards ${ }^{4}$, \& Jerry X. Mitrovica ${ }^{2}$ \\ ${ }^{1}$ Research School of Earth Sciences, Australian National University, 142 Mills Rd, Acton, ACT 200, Australia \\ ${ }^{2}$ Department of Earth \& Planetary Sciences, Harvard University, 20 Oxford Street, Cambridge, MA 02138 , USA. \\ ${ }^{3}$ Lamont-Doherty Earth Observatory, Columbia University, 61 Rte 9W, Palisades, NY 10964, USA. \\ ${ }^{4}$ Royal School of Mines, Imperial College London, South Kensington Campus, London, SW7 2AZ, UK.
}

\section{Abstract}

2 The dynamical flattening of the Earth, $H$, related to the precession constant, is a fundamental astro-geodetic parameter that 3 appears in studies of the Earth's rotation and orbital evolution. We present numerical predictions and observations of the 4 variation in $H$ over time scales ranging from tens of millions of years to decades. The geophysical processes controlling this 5 variation include solid-state convection in the rocky mantle of the Earth that drives plate tectonics, isostatic adjustments 6 due to ice age loading, and ice-ocean mass transfer linked to modern global climate change. The time dependence of $H$ is complex and non-linear, and thus, in contrast to previous suggestions, cannot be captured by a constant rate parameter.

8 Key words: Precession constant, dynamical flattening, mantle convection, glacial isostatic adjustment, climate change, 9 Earth rotation

\section{1 Introduction}

${ }_{11}$ The dynamic flattening of the Earth, $H$, is a measure of the difference between the polar moment of inertia $(C)$ and the 12 mean of the equatorial moments of inertia $(A, B)$ of the planet:

$$
H=\frac{1}{C}\left[C-\frac{1}{2}(A+B)\right]
$$

$H$ is a fundamental parameter in precession and nutation theories of the Earth, as well as a series of other rotational normal modes of widely varying frequency (e.g. Wahr, 1981; Dehant \& Capitaine, 1996; Chao, 2017). Dynamic flattening also plays an important role in a range of global geophysical studies - either explicitly or implicitly - through its connection to changes in the planetary spin rate (or, alternatively, "length-of-day" in geodesy) or dynamical form factor, $J_{2}$. For example: (1) satellite-based estimates of the secular rate of change of $J_{2}$ after $\sim 1990$ are thought to be impacted by the onset of significant polar ice sheet melting (Cox \& Chao, 2002); (2) variations in $J_{2}$ associated with ongoing, residual effects of the last ice age, as well as with tidal dissipation and other factors, combine to explain the slowing of the 
Earth's rotation rate over the past three millennia that has been estimated from ancient eclipse observations (Stephenson \& Morrison, 1984, 1995; Stephenson, 2003; Mitrovica et al., 2015); (3) perturbations in the dynamical flattening driven by mass changes arising from ice age effects and solid-state convective mantle flow alter Milankovitch (precession, obliquity) band variations in climate proxy records (Laskar et al., 1993; Forte \& Mitrovica, 1997; Mitrovica et al., 1997; Pälike \& Shackleton, 2000; Lourens et al., 2001; Morrow et al., 2012); and (4) geological measurements of the period of Earth's rotation during the Proterozoic Eon $(\sim 620 \mathrm{Ma})$ that are based on tidal rhythmites reflect long-term tidal breaking and dissipation in the Earth-Moon-Sun system (Williams, 1997), which would also be manifest as a trend in $H$.

The above discussion raises the question: Is the rate of change of dynamical flattening constant and, if not, what is the temporal structure of its variability? Burša et al. (2008) estimated that the long-term variation in $d H / d t=-8.45 \times 10^{-11} \mathrm{yr}^{-1}$ from satellite data over the period 1979-2002 (Cox \& Chao, 2002). They argue that this rate should be treated as a fundamental astro-geodetic parameter and suggest that the trend may remain valid for the past $650 \mathrm{Myr}$; this argument is based on the fact that the current rate of tidal breaking of the Earth's rotation rate would, if applied over this long time period, lead to a rotation period at $650 \mathrm{Ma}$ (henceforth "Ma" denotes "million years ago") relatively close to the geological inference of $\sim 21.8$ hours. Putting aside geophysical modeling of variations in $H$, there are a variety of reasons to be sceptical of this argument. First, as noted above, the trend in the dynamical form factor, or $d J_{2} / d t$, has varied significantly across the satellite period. For example, Roy \& Peltier (2011) estimate rates of $-3.7 \times 10^{-11} \mathrm{yr}^{-1}$ for the period 1976-1992 and $-0.9 \times 10^{-11} \mathrm{yr}^{-1}$ for 1992-2009 (equivalent values for $d H / d t$ are $-11.1 \times 10^{-11} \mathrm{yr}^{-1}$ and $-2.7 \times 10^{-11} \mathrm{yr}^{-1}$, respectively). The estimate of $d H / d t=-8.45 \times 10^{-11} \mathrm{yr}^{-1}$ in Burša et al. (2008), based on the results of Cox \& Chao (2002), is thus a time-weighted average of these two values. Second, ongoing isostatic adjustment in response to ice age loading over the last few million years dominates the pre-1992 variation in $H$, and thus any trend in the precession constant over this period cannot be constant, but will instead reflect the time scales of ice age cyclicity. Third, the current dynamic flattening of the Earth is known to exceed the form of a rotating planet in hydrostatic equilibrium by 1\% (Nakiboglu, 1982; Chambat et al., 2010). This excess flattening is driven by convective flow in the mantle and will thus vary over the timescale associated with that process, which is tens of millions of years. Finally, the present rate of tidal breaking of the Earth's rotation must be anomalously high, because a back-projection of that rate leads to the so-called "time-scale problem" of Lunar origin (i.e., the Moon's orbital radii would place it at the Roche limit only $~ 2$ billion years ago; Kaula \& Harris, 1975). The likely resolution of this problem is that ocean tidal dissipation would have been lower during the time of the Pangean supercontinent ( $\sim 340-170 \mathrm{Ma})$, and during previous supercontinent periods, with a consequent reduction in rates of change of both $H$ and Earth's rotation period during these times (Hansen, 1982).

In this article, we describe new predictions of the variation in the dynamical ellipticity over time scales ranging from tens of millions of years to centuries, based on geophysical modeling of changes in Earth's shape associated with mantle convective flow over the past 50 million years and ice mass flux across the Plio-Pleistocence glacial cycles (i.e., the past $3 \mathrm{Myr}$ ). We also map a recent, satellite-derived time series of $J_{2}$ into a variation in $H$ from 1976-2012. The predictions, 
together with the satellite derived time series, provide a measure of the natural — and human-induced — variability in

${ }_{54}$ Earth's dynamical flattening. This variability is complex, and it cannot be captured by a constant rate term.

\section{${ }_{55} 2$ Mathematical Background}

${ }_{56}$ Perturbing Equation (1), and using the fact that the trace of the inertia tensor is invariant during non-uniform deformation $57 \quad$ (e.g. Rochester \& Smylie, 1974), i.e.,

$$
\delta A+\delta B+\delta C=0
$$

${ }_{58} \quad$ yields the following expression for the variation in $H$

$$
\delta H=\left[\frac{3}{2}-H\right] \frac{\delta C}{C} .
$$

60 expression can be approximated as

$$
\delta H=\frac{3}{2} \frac{\delta C}{C} .
$$

61 The dynamical form factor, $J_{2}$, is defined as

$$
J_{2}=\frac{1}{M_{e} a^{2}}\left[C-\frac{1}{2}(A+B)\right],
$$

where $M_{e}$ and $a$ are the mass and radius of the Earth, respectively. Combining equations (1) and (5) yields the following relationship

$$
H=\frac{M_{e} a^{2}}{C} J_{2} .
$$

Taking the first variation of this expression, and once again using the fact that $H<<1$, yields

$$
\delta H=\frac{M_{e} a^{2}}{C} \delta J_{2} .
$$

Since $C \sim \frac{1}{3} M_{e} a^{2}$, the scaling factor on the right-hand side of Equation (7) is $\sim 3$. This simple relationship was applied to relate expressions for $d J_{2} / d t$ and $d H / d t$ that were used in Section 1.

In the results below, we will consider predictions and observations of the relative perturbation in the dynamic flattening, $\delta H / H_{0}$, where $H_{0}$ is the present-day value (0.003274) and the perturbation $\delta$ is defined relative to this value (Figure 1 ). As a guide to interpreting the impact of such signals, if a dynamic ellipticity of value $H^{\prime}$ is connected to an orbital frequency (for example, of precession or obliquity variations) of $f^{\prime}$, then, in the absence of resonance effects, the perturbation $\delta H / H^{\prime}$ would yield a proportional perturbation in the associated frequency of $f^{\prime} \delta H / H^{\prime}$ (Laskar et al., 1993; Williams, 1994). 


\section{Results}

Plate tectonics is driven by thermochemical convection within the Earth's mantle, a process that also leads to perturbations in the shape of the solid surface, core-mantle boundary, and gravitational field of the planet on a wide range of spatial scales. In the 1980s, global geophysical research focused on numerical and theoretical modeling of the process using constraints from satellite-derived estimates of Earth's long-wavelength gravity field (e.g., Richards \& Hager, 1984; Ricard et al., 1984; Hager et al., 1985). These efforts combined tomographic models of seismic velocity variations in the mantle with experimental constraints from mineral physics on the mapping between these velocities and density (or, equivalently, buoyancy), with the goal of constraining the depth-dependent variation of mantle viscosity. While this approach provides invaluable insights on mantle dynamics, trade-offs between mantle buoyancy and viscosity render results subject to considerable uncertainty (Thoraval \& Richards, 1997). Subsequent work therefore extended these studies to consider a wider range of present-day observations, including plate velocities, perturbations to surface topography, and excess ellipticity of the core-mantle boundary as inferred from the period of the Earth's free core nutation (e.g. Forte \& Peltier, 1987; Lithgow-Bertelloni \& Richards, 1998; Gurnis et al., 2000; Forte \& Mitrovica, 2001; Simmons et al., 2006).

A number of studies have extended the present-day snapshot of mantle dynamics, the focus of the above analyses, to model the time history of the system. These analyses were generally based on "backward advection" of the governing field equations under the caveat that thermal diffusion is treated as negligible, since it is not temporally reversible in a unique sense and is not tractable due to numerical instabilities (Steinberger \& O'Connell, 1997; Conrad \& Gurnis, 2003; Moucha et al., 2008). A major limitation of this approach is that it produces transient behaviour within the thermal boundary layers (regions at the base and top of the convecting mantle, which are dominated by conductive heat transport), resulting in model simulations undergoing an initial jump prior to reaching steady-state; this jump contaminates the most recent period of model evolution. These issues are avoided in more sophisticated adjoint treatments that solve the full field equations in a forward sense and therefore rigorously incorporate thermal diffusion (e.g., Bunge et al., 2003; Ismail-Zadeh et al., 2004; Zhou \& Liu, 2017; Li et al., 2017; Price \& Davies, 2018; Ghelichkhan \& Bunge, 2018).

Here, we adopt the adjoint methodology of Ghelichkhan \& Bunge (2016) to track relative changes in the dynamical ellipticity driven by mantle convection over the past $50 \mathrm{Myr}$ (Figure 1A). Details of the calculation are provided in the Appendix. Our simulation yields a perturbation in the magnitude of $H$ of order $0.1 \%$ since $50 \mathrm{Ma}$, with an increase in dynamic flattening until $15 \mathrm{Ma}$, followed by a decrease of comparable magnitude in the subsequent $15 \mathrm{Myr}$ (Figure 1A).

Our investigation of the evolving mantle heterogeneity in the adjoint model indicates that the increase in $\delta H / H$ from 50 Ma to $20 \mathrm{Ma}$ is driven by an increase in the amplitude of long-wavelength density anomalies at the base of the upper mantle (the so-called transition zone) and the base of the lower mantle (i.e., above the fluid outer core). The subsequent change in trend reflects a progressive weakening of the transition zone signal after $20 \mathrm{Ma}$.

This variation in $H$ is significantly smaller than predicted by a previous backward advection simulation (Forte \& 
Mitrovica, 1997), and it has significant implications for the stability of Earth's precession and obliquity parameters. In particular, Laskar et al. (1993) has shown that if the dynamic flattening were perturbed downward by $\sim 0.2 \%$ relative to the present day value, these parameters would experience a non-linear perturbation due to a passage through the $s_{6}-g_{6}+g_{5}$ resonance that is associated with perihelion of Jupiter and Saturn and the node of Saturn. We conclude that such a passage is unlikely to have occurred over the past 50 Myr (c.f. Forte \& Mitrovica, 1997).

Next, we turn to variability in the dynamical ellipticity associated with ice age dynamics over the past $3 \mathrm{Myr}$. Over this period, the Earth was subject to glacial cycles of increasing magnitude, and an obliquity-paced periodicity of $\sim 40$ kyr until $\sim 800 \mathrm{ka}$, followed by the so-called "Mid-Pleistocene transition" to cycles of period 100 kyr (Lisiecki \& Raymo, 2005). The last such cycle occurred from $\sim 120-6 \mathrm{ka}$, with the Last Glacial Maximum reached at $26 \mathrm{ka}$, and it involved a mass flux equivalent to $\sim 130 \mathrm{~m}$ of global average sea level change (Austermann et al., 2013). Our prediction of perturbations to the dynamical flattening (Figure 1B) is generated using a theory of ice age dynamics that involves a gravitationally self-consistent treatment of sea level changes constrained to conserve the total (ice plus ocean) surface mass (Kendall et al., 2005) and it requires, on input, models for the radial profile of mantle viscosity and the full space-time geometry of ice mass changes. For the former, we adopt the same viscosity model used in our convection simulation to generate Figure 1A, and for the latter, we use the ice history developed by Raymo et al. (2011).

Since the Earth is currently in an interglacial period, with high-latitude glaciation near a minimum, the mean perturbation of $H$ relative to present day represents a reduction in the flattening of $0.12 \%$. Over the same period, the convection-induced perturbation to $H$ reaches $0.015 \%$ of the present-day value, and thus ice age dynamics dominate the perturbation in dynamical flattening across this $3 \mathrm{Myr}$ time scale. The temporal variability in Figure $1 \mathrm{~B}$ reflects the history of forcing, with the above-noted transition in the period of cyclicity and a general change in the magnitude of variability at $\sim 800 \mathrm{ka}$. Across the current interglacial (i.e., since $6 \mathrm{ka}$ ), the polar regions of the Earth are continuing to rebound from subsidence associated with 26-6 ka ice unloading, and this process is reflected in the gradual reduction in flattening that persists to the present day.

Finally, we turn our attention to recent variations in the dynamical flattening on decadal time scales. Figure 1C shows the observed change in $H$ across the satellite period, relative to 2012, derived from the results of Cheng et al. (2013). As discussed earlier, a change in the trend of the $\delta H / H_{0}$ time series, or equivalently $J_{2}$ from Equation (5), took place around the year 1990. Prior to that date, the trend is dominated by the above-noted reduction in oblateness (and polar moment of inertia) since $\sim 6 \mathrm{ka}$ that is driven by ongoing effects of the ice age. This trend continues after 1990, but the onset of significant modern melting of ice sheets at that time contributes to an increase in oblateness (as ice melts near the poles and mass redistributes toward lower latitudes), resulting in a net signal that is characterized by a reduced trend (i.e., the magnitude of $d H / d t$ and $d J_{2} / d t$ decreases).

In more quantitative terms, the rate of change in $\delta H / H_{0}$ prior to 1990 is $-3.4 \times 10^{-8} \mathrm{yr}^{-1}$, and it decreases in magnitude by approximately a factor of four to $-0.8 \times 10^{-8} \mathrm{yr}^{-1}$ in the period 1990-2012. The ice age calculation of Figure 1B 
predicts a contribution to the present-day rate of change of $\delta H / H_{0}$ of approximately $-5.1 \times 10^{-8} \mathrm{yr}^{-1}$, and correcting the two observed rates for this signal yields residuals of $\sim 1.7 \times 10^{-8} \mathrm{yr}^{-1}$ and $\sim 4.3 \times 10^{-8} \mathrm{yr}^{-1}$, respectively. In the earlier period, 1976-1990, the remaining contributor to the signal is associated with melting of glaciers driven by global climate change. Mitrovica et al. (2015) estimated the rate of change of $J_{2}$ due to this glacier melting as $\sim 2.0 \pm 0.3 \times 10^{-11} \mathrm{yr}^{-1}$ this converts to a rate of change in $\delta H / H_{0}$ of $\sim 1.8 \times 10^{-8} \mathrm{yr}^{-1}$, a value which is in agreement with the (observed minus ice age-corrected) residual cited above $\left(\sim 1.7 \times 10^{-8} \mathrm{yr}^{-1}\right)$. In the period after 1990, the larger ice age-corrected signal $\left(4.3 \times 10^{-8} \mathrm{yr}^{-1}\right)$ reflects the onset of major melting from the polar ice sheets (Cox \& Chao, 2002; Cheng et al., 2013). The best fit linear form across the full time series, i.e., 1976-2012, is characterized by a rate of change of $\delta H / H_{0}$ of $\sim-2.1 \times 10^{-8} \mathrm{yr}^{-1}$.

The calculations in Figure 1, since they are based on Equation (2), do not include the impact on the inertia tensor of a uniform, degree-0 deformation of the Earth. If we included this spatially uniform signal in the theory, Equation (3) would be revised to

$$
\delta H=\left[\frac{3}{2}-H\left(1+\frac{\delta C_{(0,0)}}{\delta C_{(2,0)}}\right)\right] \frac{\delta C_{(2,0)}}{C},
$$

where the subscripts denote the spherical harmonic degree and order of the structure contributing to the inertia perturbation. In our calculations of perturbations in $\delta H / H$ due to ice mass changes (Figure 1B), we include complementary sea level changes and the total mass of the surface load is conserved (i.e., it has no degree-0 component). The same must be true for the processes responsible for the observations that form the basis of Figure $1 \mathrm{C}$. Thus, in these cases, there is no degree-0 deformation, $\delta C_{(0,0)}=0$, and the above expression collapses to that in Equation (3). While our calculations of the perturbation to $\delta H / H$ driven by mantle convection adopt a compressible flow model, any changes in the volume of the Earth are negligible. However, these calculations do not account for secular cooling and thermal contraction of the Earth. Estimates of this latter process suggest that the reduction in Earth radius over the past 50 Myr due to thermal contraction has been $\sim 80 \mathrm{~m}$ (Tsuchiya et al., 2013). Using this value and the results in Figure $1 \mathrm{~A}$ yields an estimate of $\delta C_{(0,0)} / \delta C_{(2,0)} \sim 5$, and therefore $H\left(1+\delta C_{(0,0)} / \delta C_{(2,0)}\right) \sim 6 H$. This value is of order $1 \%$ of the leading term of $\frac{3}{2}$ in Equation (8), and neglecting it in adopting Equation (4) remains justified.

The results in Figure 1 do not include the impact on the dynamical flattening of an additional process mentioned in the introductory section, namely, tidal dissipation. The present level of tidal dissipation is slowing the Earth's rotation at a rate of $(d \Omega / d t) / \Omega=8.8 \times 10^{-18} \mathrm{~s}^{-1}$ (e.g. Quinn et al., 1991) and the dynamical flattening will be approximately proportional to $\Omega^{2}$. While the variation of tidal dissipation over time is uncertain, any effort to estimate the total change in dynamical flattening from all geophysical processes must include this contribution. 


\section{Final Remarks}

The dynamical flattening of the Earth, a parameter associated with the precession constant, plays an important role in a wide range of applications in astronomy, geodesy and geophysics, including astronomical observations of nutations, investigations of the stability of the orbital elements (precession, obliquity) controlling Milankovitch forcing of ice age climate, and the evolution of the Earth-Moon-Sun system over billion-year time scales. Burša et al. (2008) highlighted the importance of recognizing the time dependence in the precession constant within astronomical analyses. However, they suggested that the variation in $H$ could be captured by a constant rate term computed by fitting a linear form through a satellite time series of $J_{2}$ extending from 1979-2002 - they derived a value for $d H / d t$ of $-8.45 \times 10^{-11} \mathrm{yr}^{-1}$, or equivalently a rate of change of $\delta H / H_{0}$ of $\sim 2.6 \times 10^{-8} \mathrm{yr}^{-1}-$ and advocated that the rate be adopted as a fundamental astro-geodetic parameter. In contrast to this view, we have shown in Figure 1 that time dependence of the dynamic ellipticity is highly non-linear, even when considering only the last 40 years of satellite-based measurements (Figure 1C). The full complexity of the time series of $\delta H / H_{0}$ in Figure 1 reflects the suite of geophysical processes that perturb the Earth's flattening, including mantle convection, ice age dynamics, and modern global climate change.

\section{Acknowledgements}

SG is indebted to D.R. Davies for guidance and support. We acknowledge funding and support from Harvard University (JJF, JXM), National Aeronautics and Space Administration grant NNX17AE17G (MJH, JXM), the American Chemical Society Petroleum Research Fund grant 59062-DNI8 (MJH), Imperial College Research Fellowship scheme and the Schmidt Science Fellows program (FDR)

\section{Appendix}

Time-evolution in our adjoint treatment (Ghelichkhan \& Bunge, 2016) is constrained by assimilating a history of plate motions (Young et al., 2019), and the initial buoyancy field (i.e., at $50 \mathrm{Ma}$ ) is iteratively optimized through comparison of the final, present-day buoyancy field predicted by the flow model with the buoyancy field inferred from seismic tomography. This procedure typically converges after 12-15 iterations.

Two other fields need to be prescribed in this procedure, the radial viscosity structure used in the flow calculation and the present-day mantle buoyancy field to which the prediction of the flow model at the present day is compared. We consider each, in turn. All other material parameters and boundary conditions are adopted from Colli et al. (2018).

We use a radial viscosity profile derived from a joint inversion of data related to mantle convection and ice age dynamics (Mitrovica \& Forte, 2004). The viscosity model, which we also adopt in the ice age calculations described in the main text, is characterized by a three order of magnitude increase in viscosity from the shallow mantle beneath 
the lithosphere $\left(10^{20} \mathrm{~Pa} \mathrm{~s}\right)$ to $2000 \mathrm{~km}$ depth $\left(10^{23} \mathrm{~Pa} \mathrm{~s}\right)$, followed by a reduction of comparable magnitude toward the core-mantle-boundary.

To construct the present-day mantle buoyancy field, we use lower mantle shear wave velocities from the recent tomography model LLNL-G3D (Simmons et al., 2012). Upper mantle velocity structure is prescribed from the higher resolution surface wave tomography model SL2013sv (Schaeffer \& Lebedev, 2013), smoothly blended into the deeper mantle model over the depth range 250-350 km. To convert seismic velocities into density, we first calculate anharmonic velocities and densities as a function of pressure and temperature for a pyrolitic mantle composition using the thermodynamic database of Stixrude \& Lithgow-Bertelloni (2011) and the Perple_X Gibbs free-energy minimisation software (Connolly, 2005). Next, anharmonic velocities are corrected for anelasticity using the Q5 attenuation model of Cammarano et al. (2003), adopting the solidus of Hirschmann (2000) in the upper $~ 250 \mathrm{~km}$ and Andrault et al. (2011) in the deeper mantle. Tomographically inferred velocity variations as a function of depth are then used to query the resulting lookup table and extract corresponding values of density. To prevent the continental lithosphere from actively participating in convection, densities within the lithosphere are set to the radial average using the lithosphere-asthenosphere boundary map of Hoggard et al. (2020).

Once the temporal evolution of the mantle flow field has been successfully reconstructed, we calculate the time history of dynamic ellipticity, $H$. For this purpose, we solve the governing, coupled system of Stokes and Poisson's equations using an instantaneous flow methodology that includes the effects of self-gravitation and compressiblity and assumes a free-slip (no tangential stress) boundary condition (Corrieu et al., 1995).

\section{References}

Andrault, D., Bolfan-Casanova, N., Nigro, G. L., Bouhifd, M. A., Garbarino, G., \& Mezouar, M., 2011. Solidus and liquidus profiles of chondritic mantle: Implication for melting of the Earth across its history, Earth and Planetary Science Letters, 304, $251-259$.

Austermann, J., Mitrovica, J. X., Latychev, K., \& Milne, G. A., 2013. Barbados-based estimate of ice volume at Last Glacial Maximum affected by subducted plate, Nature Geoscience, 6, 553-557.

Bunge, H.-P., Hagelberg, C. R., \& Travis, B. J., 2003. Mantle circulation models with variational data assimilation: Inferring past mantle flow and structure from plate motion histories and seismic tomography, Geophysical Journal International, 152, $280-301$.

Burša, M., Groten, E., \& Šíma, Z., 2008. Steady change in flattening of the Earth: The precession constant and its long-term variation, The Astronomical Journal, 135, 1021-1023.

Cammarano, F., Goes, S., Vacher, P., \& Giardini, D., 2003. Inferring upper-mantle temperatures from seismic velocities, Physics of the Earth and Planetary Interiors, 138, 197-222.

Chambat, F., Ricard, Y., \& Valette, B., 2010. Flattening of the Earth: Further from hydrostaticity than previously estimated, Geophysical Journal International, 183, 727-732.

Chao, B. F., 2017. On rotational normal modes of the Earth: Resonance, excitation, convolution, deconvolution and all that, Geodesy and Geodynamics, 8, 371-376.

Cheng, M., Tapley, B. D., \& Ries, J. C., 2013. Deceleration in the Earth's oblateness, Journal of Geophysical Research: Solid Earth, 118, 740-747.

Colli, L., Ghelichkhan, S., Bunge, H. P., \& Oeser, J., 2018. Retrodictions of Mid Paleogene mantle flow and dynamic topography in the Atlantic region from compressible high resolution adjoint mantle convection models: Sensitivity to deep mantle viscosity and tomographic input model, Gondwana Research, 53, 252-272.

Connolly, J. A., 2005. Computation of phase equilibria by linear programming: A tool for geodynamic modeling and its application to subduction zone decarbonation, Earth and Planetary Science Letters, 236, 524-541. 
Conrad, C. P. \& Gurnis, M., 2003. Seismic tomography, surface uplift, and the breakup of Gondwanaland: Integrating mantle convection backwards in time, Geochemistry, Geophysics, Geosystems, 4(3).

Corrieu, V., Thoraval, C., \& Ricard, Y., 1995. Mantle dynamics and geoid Green functions, Geophysical Journal International, 120, $516-523$.

Cox, C. M. \& Chao, B. F., 2002. Detection of a large-scale mass redistribution in the terrestrial system since 1998, Science, 297, 831-833.

Dehant, V. \& Capitaine, N., 1996. On the precession constant: Values and constraints on the dynamical ellipticity; Link with Oppolzer terms and tilt-over-mode, Celestial Mechanics and Dynamical Astronomy, 65, 439-458.

Forte, A. M. \& Mitrovica, J. X., 1997. A resonance in the Earth's obliquity and precession over the past 20 Myr driven by mantle convection, Nature, 390, 676-680.

Forte, A. M. \& Mitrovica, J. X., 2001. Deep-mantle high-viscosity flow and thermochemical structure inferred from seismic and geodynamic data., Nature, 410, 1049-1056.

Forte, A. M. \& Peltier, W. R., 1987. Plate tectonics and aspherical Earth structure: The importance of poloidal- toroidal coupling, Journal of Geophysical Research, 92(B5), 3645-3679.

Ghelichkhan, S. \& Bunge, H.-P., 2016. The compressible adjoint equations in geodynamics: Derivation and numerical assessment, International Journal on Geomathematics, 7, 1-30.

Ghelichkhan, S. \& Bunge, H. P., 2018. The adjoint equations for thermochemical compressible mantle convection: Derivation and verification by twin experiments, Proceedings of the Royal Society A, 474(20180329).

Gurnis, M., Mitrovica, J. X., Ritsema, J., \& van Heijst, H.-J., 2000. Constraining mantle density structure using geological evidence of surface uplift rates: The case of the African Superplume, Geochemistry, Geophysics, Geosystems, 1(1020).

Hager, B. H., Clayton, R. W., \& Richards, M. A., 1985. Lower mantle heterogeneity, dynamic topography and the geoid, Nature, 313, $541-545$.

Hansen, K. S., 1982. Secular effects of oceanic tidal dissipation on the Moon's orbit and the Earth's rotation, Reviews of Geophysics, 20(3), 457-480.

Hirschmann, M. M., 2000. Mantle solidus: Experimental constraints and the effects of peridotite composition, Geochemistry Geophysics Geosystems, 1(10).

Hoggard, M. J., Czarnota, K., Richards, F. D., Huston, D. L., Jaques, A. L., \& Ghelichkhan, S., 2020. Global distribution of sediment-hosted metals conrolled by craton edge stability, Nature Geoscience, 13, 504-510.

Ismail-Zadeh, A., Schubert, G., Tsepelev, I., \& Korotkii, A., 2004. Inverse problem of thermal convection: Numerical approach and application to mantle plume restoration, Physics of the Earth and Planetary Interiors, 145, 99-114.

Kaula, W. M. \& Harris, A. W., 1975. Dynamics of lunar origin and orbital evolution, Reviews of Geophysics and Space Physics, 13(2), 363-371.

Kendall, R. A., Mitrovica, J. X., \& Milne, G. A., 2005. On post-glacial sea level - II. Numerical formulation and comparative results on spherically symmetric models, Geophysical Journal International, 161, 679-706.

Laskar, J., Joutel, F., \& Boudin, F., 1993. Orbital, precessional, and insolation quantities for the Earth from $-20 \mathrm{Myr}$ to $+10 \mathrm{Myr}$, Astronomy and Astrophysics, 270, 522-533.

Li, D., Gurnis, M., \& Stadler, G., 2017. Towards adjoint-based inversion of time-dependent mantle convection with nonlinear viscosity, Geophysical Journal International, 209, 86-105.

Lisiecki, L. E. \& Raymo, M. E., 2005. A Pliocene-Pleistocene stack of 57 globally distributed benthic $\delta 18$ O records, Paleoceanography, 20(PA1003).

Lithgow-Bertelloni, C. \& Richards, M. A., 1998. The dynamics of Cenozoic and Mesozoic plate motions, Reviews of Geophysics, 36(1), $27-78$.

Lourens, L. J., Wehausen, R., \& Brumsack, H. J., 2001. Geological constraints on tidal dissipation and dynamical ellipticity of the Earth over the past three million years, Nature, 409, 1029-1033.

Mitrovica, J. X. \& Forte, A. M., 2004. A new inference of mantle viscosity based upon joint inversion of convection and glacial isostatic adjustment data, Earth and Planetary Science Letters, 225, 177-189.

Mitrovica, J. X., Forte, A. M., \& Pan, R., 1997. Glaciation-induced variations in the Earth's precession frequency, obliquity and insolation over the last 2.6 Ma, Geophysical Journal International, 128, 270-284.

Mitrovica, J. X., Hay, C. C., Morrow, E., Kopp, R. E., Dumberry, M., \& Stanley, S., 2015. Reconciling past changes in Earth's rotation with 20th century global sea-level rise: Resolving Munk's enigma, Science Advances, 1(e1500679).

Morrow, E., Mitrovica, J. X., Forte, A. M., Glišović, P., \& Huybers, P., 2012. An enigma in estimates of the Earth’s dynamic ellipticity, Geophysical Journal International, 191, 1129-1134.

Moucha, R., Forte, A. M., Mitrovica, J. X., Rowley, D. B., Quéré, S., Simmons, N. A., \& Grand, S. P., 2008. Dynamic topography and long-term sea-level variations: There is no such thing as a stable continental platform, Earth and Planetary Science Letters, 271, 101-108. 


\section{Manuscript submitted to Icarus (Letter) on 30th August 2020}

Nakiboglu, S. M., 1982. Hydrostatic theory of the Earth and its mechanical implications, Physics of the Earth and Planetary Interiors, 28, $302-311$.

Pälike, H. \& Shackleton, N. J., 2000. Constraints on astronomical parameters from the geological record for the last 25 Myr, Earth and Planetary Science Letters, 182, 1-14.

Price, M. G. \& Davies, J. H., 2018. Profiling the robustness, efficiency and limits of the forward-adjoint method for 3-D mantle convection modelling, Geophysical Journal International, 212, 1450-1462.

Quinn, T. R., Tremaine, S., \& Duncan, M., 1991. A three million year integration of the Earth's orbit, The Astronomical Journal, 101(6), $2287-2305$.

Raymo, M. E., Mitrovica, J. X., O’Leary, M. J., Deconto, R. M., \& Hearty, P. J., 2011. Departures from eustasy in Pliocene sea-level records, Nature Geoscience, 4, 328-332.

Ricard, Y., Fleitout, L., \& Froidevaux, C., 1984. Geoid heights and lithospheric stresses for a dynamic Earth, Annales Geophysicae, 2, $267-286$.

Richards, M. A. \& Hager, B. H., 1984. Geoid anomalies in a dynamic Earth, Journal of Geophysical Research, 89(B7), 5987-6002.

Rochester, M. G. \& Smylie, D. E., 1974. On changes in the trace of the Earth's inertia tensor, Journal of Geophysical Research, 79(32), $4948-4951$.

Roy, K. \& Peltier, W. R., 2011. GRACE era secular trends in Earth rotation parameters: A global scale impact of the global warming process?, Geophysical Research Letters, 38(L10306).

Schaeffer, A. J. \& Lebedev, S., 2013. Global shear speed structure of the upper mantle and transition zone, Geophysical Journal International, 194, 417-449.

Simmons, N. A., Forte, A. M., \& Grand, S. P., 2006. Constraining mantle flow with seismic and geodynamic data: A joint approach, Earth and Planetary Science Letters, 246, 109-124.

Simmons, N. A., Myers, S. C., Johannesson, G., \& Matzel, E., 2012. LLNL-G3Dv3: Global P wave tomography model for improved regional and teleseismic travel time prediction, Journal of Geophysical Research: Solid Earth, 117(B10302).

Steinberger, B. \& O'Connell, R. J., 1997. Changes of the Earth's rotation axis owing to advection of mantle density heterogeneities, Nature, 387, $169-173$.

Stephenson, F. R., 2003. Historical eclipses and Earth's rotation, Astronomy and Geophysics, 44(2), 22-27.

Stephenson, F. R. \& Morrison, L. V., 1984. Long-term changes in the rotation of the Earth: 700 B.C. to A.D. 1980, Philosophical Transactions of the Royal Society of London A, 313, 47-70.

Stephenson, F. R. \& Morrison, L. V., 1995. Long-term fluctuations in the Earth's rotation: 700 BC to AD 1990, Philosophical Transactions of the Royal Society of London A, 351, 165-202.

Stixrude, L. \& Lithgow-Bertelloni, C., 2011. Thermodynamics of mantle minerals - II. Phase equilibria, Geophysical Journal International, 184, $1180-1213$.

Thoraval, C. \& Richards, M. A., 1997. The geoid constraint in global geodynamics: Viscosity structure, mantle heterogeneity models and boundary conditions, Geophysical Journal International, 131, 1-8.

Tsuchiya, T., Kawai, K., \& Maruyama, S., 2013. Expanding-contracting Earth, Geoscience Frontiers, 4, 341-347.

Wahr, J. M., 1981. Body tides on an elliptical, rotating, elastic and oceanless earth, Geophysical Journal of the Royal Astronomical Society, 64, 677-703.

Williams, G. E., 1997. Precambrian length of day and the validity of tidal rhythmite paleotidal values, Geophysical Research Letters, 24(4), $421-424$.

Williams, J. G., 1994. Contributions to the Earth's obliquity rate, precession, and nutation, Astron. J., 108, 711-724.

Young, A., Flament, N., Maloney, K., Williams, S., Matthews, K., Zahirovic, S., \& Müller, R. D., 2019. Global kinematics of tectonic plates and subduction zones since the late Paleozoic Era, Geoscience Frontiers, 10, 989-1013.

Zhou, Q. \& Liu, L., 2017. A hybrid approach to data assimilation for reconstructing the evolution of mantle dynamics, Geochemistry, Geophysics, Geosystems, 18, 3854-3868. 

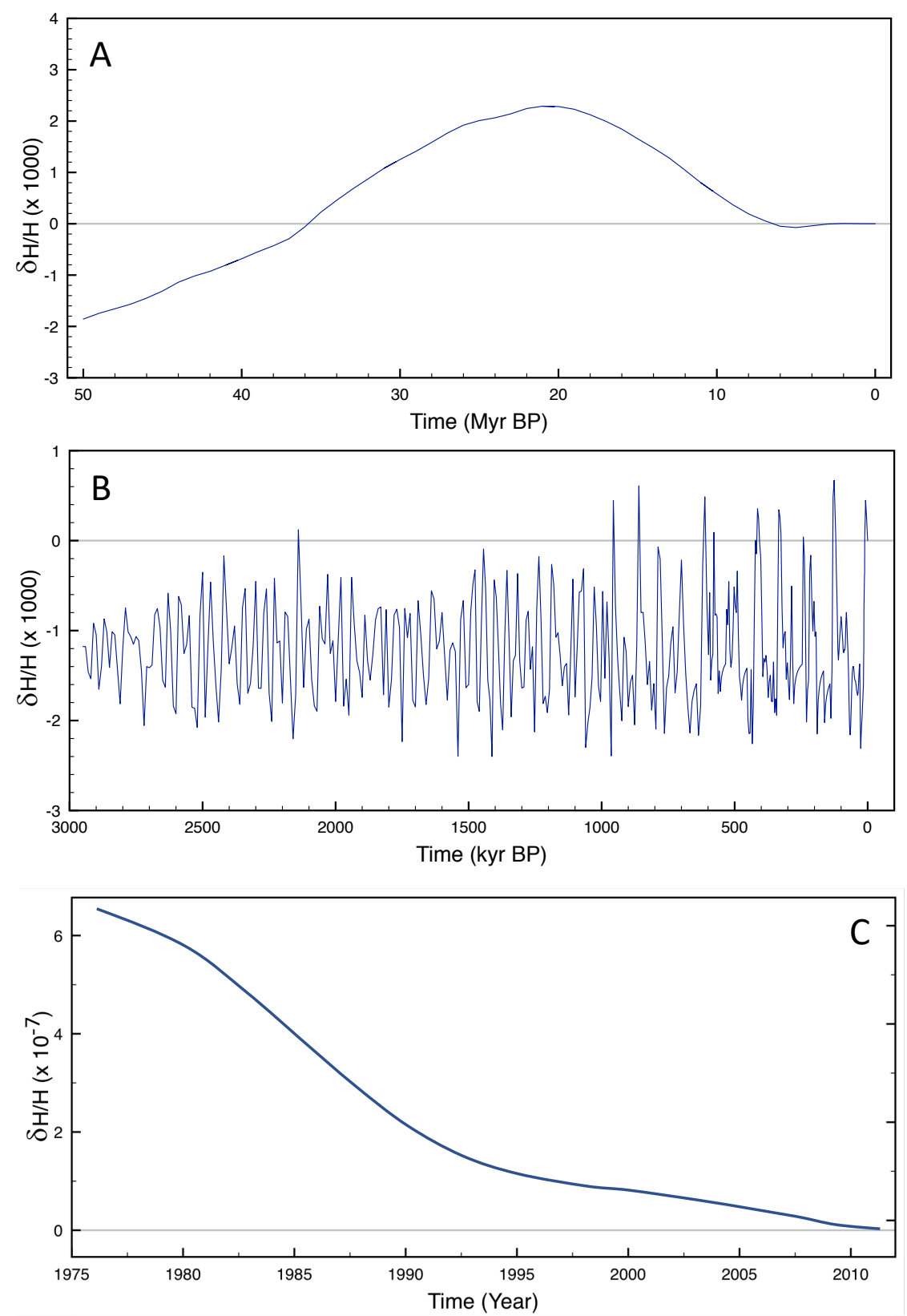

Figure 1: (A) Relative perturbation in the precession constant of the Earth predicted using the adjoint reconstruction of mantle convective flow over the past $50 \mathrm{Myr}$ (see text). (B) As in (A), except for a reconstruction of changes in the precession constant since 3 Ma predicted from a simulation of ice age dynamics alone (see text). The numerical predictions in frames (A) and (B) are both based on a common model of the Earth's radial viscosity profile that was derived by simultaneously inverting a large suite of global geophysical data related to mantle convection and ice age dynamics (Mitrovica \& Forte, 2004; see text). (C) Time series of the relative perturbation in the precession constant estimated from satellite altimetry records since 1975. The time series is computed from the results of Cheng et al. (2013). All results are plotted relative to the present day value, or in the case of frame (C), 2012. 